\title{
On a Mixed Problem for Hyperbolic Equations with Discontinuous Boundary Conditions
}

By

\author{
Kazunari HAYASHIDA*
}

1. Mixed problems for hyperbolic equations have been studied by many authors. For variable coefficients the powerful tool is the theorem of Hille-Yosida $([3],[4],[5],[7])$. In this case the mixed problems have been treated completely by Mizohata [5] and Ikawa [3].

On the other hand Čehlov $[2]$ has shown the existence of a weak solution for mixed problems under discontinuous boundary conditions. $\mathrm{He}$ has imposed the assumption that the space domain is a half space and the equation is the wave equation. His method is the Fourier-Laplace transformation.

In this note we consider a mixed problem under discontinuous boundary conditions of Dirichlet or Neumann type. We proceed mainly along the lines of $[3]$ and $[5]$.

2. Let $\Omega$ be a bounded domain in the $n$-dimensional Euclidean space $R^{n}$ with boundary $\partial \Omega$ of class $C^{\infty}$. We assume that $\partial \Omega$ consists of two measurable sets $\partial_{1} \Omega$ and $\partial_{2} \Omega$ having no common points. Further let us assume that

$$
\partial_{2} \Omega \cap \overline{\partial_{1} \Omega}=\phi
$$

We set

and

$$
\begin{aligned}
(u, v)_{k} & =\int_{\Omega} \sum_{|\alpha| \leq k} D^{\alpha} u \overline{D^{\alpha} v} d x, \\
\|u\|_{k}^{2} & =(u, u)_{k}
\end{aligned}
$$

Received November 12, 1970.

Communicated by S. Mizohata.

* Department of Mathematics, Nagoya University, Chikusa-ku, Nagoya, Japan. 


$$
(u, v)=(u, v)_{0}, \quad\|u\|=\|u\|_{0} .
$$

Let us denote by $H^{k}(\Omega)$ the Sobolev space with norm \|\|$_{k}$ and by $K(\Omega)$ the completion of all $u$ each of which belongs to $C^{\infty}(\bar{\Omega})$ and vanishes in a neighborhood of $\partial_{1} \Omega$ with $H^{1}(\Omega)$ norm.

Consider the elliptic operator $L$ of second order on $\bar{\Omega} \times[0, T]$ :

$$
L=-\sum_{i, j} \frac{\partial}{\partial x_{i}}\left(a_{i j}(x) \frac{\partial}{\partial x_{j}}\right)+\sum_{i} b_{i}(x, t) \frac{\partial}{\partial x_{i}}+c(x, t),
$$

where the coefficients are all in $C^{\infty}(\bar{\Omega} \times[0, T])$. We assume that $a_{i j}(x)$ $\left(=a_{j i}(x)\right)$ are real valued and positive definite on $\bar{\Omega}$ and we consider the following equation for real valued $h_{i}(x), h(x) \in C^{\circ}(\bar{\Omega})$ :

$$
\frac{\partial^{2}}{\partial t^{2}} u+L u+\left(2 \sum_{i} h_{i}(x) \frac{\partial}{\partial x_{i}}+h(x)\right) \frac{\partial}{\partial t} u=f .
$$

Let us impose the following boundary condition

$$
\begin{gathered}
B_{1} u(x, t)=u(x, t)=0 \quad \text { on } \quad \partial_{1} \Omega \times[0, T], \\
B_{2} u(x, t)=\left\{\frac{d}{d n}-<h, \gamma>\frac{\partial}{\partial t}+\sigma(x, t)\right\} u(x, t)=0 \\
\text { on } \partial_{2} \Omega \times[0, T],
\end{gathered}
$$

where

$$
\begin{aligned}
& \frac{d}{d n}=\sum_{i, j} a_{i j}(x) \cos \left(\nu, x_{j}\right) \frac{\partial}{\partial x_{i}} \quad(\nu \text { is the exterior mormal vector), } \\
& <h, \gamma>=\sum_{i} h_{i}(x) \cos \left(\nu, x_{i}\right)
\end{aligned}
$$

and $\sigma(x)$ is $C^{\infty}$ on $\partial_{2} \Omega$. The equation (2.3) has been considered in [3] and [5] under the boundary condition $B_{1} u=0$ or $B_{2} u=0$ on $\partial \Omega \times[0, T]$.

Now we define the boundary condition (2.5) in the weak sense as follows :

Definition 2.1. Let $u(., t)$ be in $H^{1}(\Omega)$ and $(L u)(., t)$ be in $L^{2}(\Omega)$ for $0 \leq t \leq T$. Further we assume that $u$ is in $\mathscr{E}_{t}^{1}\left(H^{1}(\Omega)\right)[0, T] .^{1)}$ Then $u$ is said to satisfy the boundary condition (2.5) weakly on $\partial_{2} \Omega \times[0, T]$,

1) For the Banach space $E$ the letter $\mathscr{E}_{t}^{k}(E)[0, T]$ means the set of $E$-valued functions which are $k$-times continuously differentiable in $0 \leqq t \leqq T$. 
if the following equality holds on $[0, T]$;

$$
\begin{gathered}
\left(\left\{-\frac{\partial}{\partial x_{i}}\left(a_{i j} \frac{\partial}{\partial x_{j}}\right)\right\} u, \varphi\right)=\sum_{i, j}\left(a_{i j^{j}} \frac{\partial u}{\partial x_{i}}, \frac{\partial \varphi}{\partial x_{j}}\right) \\
+\int_{\partial_{2} \Omega}\left(\sigma u-<h, \gamma>\frac{\partial u}{\partial t}\right) \bar{\varphi} d S
\end{gathered}
$$

for any $\varphi \in K(\Omega)$.

In addition we define the boundary condition for vector functions as follows.

Definition 2.2. Let $U=\{u, v\}$ be in $H^{1}(\Omega) \lll H^{1}(\Omega)$ and $L u$ be in $L^{2}(\Omega)$. Then $U$ is said to satisfy the boundary condition $\left(B_{2}\right)$ on $\partial_{2} \Omega$, if the following equality holds for any $\varphi \in K(\Omega)$;

$$
\begin{gathered}
\left(\left\{-\frac{\partial}{\partial x_{i}}\left(a_{i j} \frac{\partial}{\partial x_{j}}\right) u, \varphi\right)=\sum_{i, j}\left(a_{i j} \frac{\partial u}{\partial x_{i}},-\frac{\partial \varphi}{\partial x_{j}}\right)\right. \\
+\int_{\partial_{2} \Omega}(\sigma u-<h, \gamma>v) \bar{\varphi} d S .
\end{gathered}
$$

In this note we shall prove the following theorems, where we assume that $f(x, t) \in \mathscr{E}_{t}^{0}(K(\Omega))[0, T]$ and $f(x, 0)$ has compact support in $\Omega$.

Theorem 1. Suppose that $u_{0}(x), v_{0}(x) \in K(\Omega), L u_{0} \in L^{2}(\Omega)$ and $\left\{u_{0}, v_{0}\right\}$ satisfies the boundary condition $\left(B_{2}\right)$ on $\partial_{2} \Omega$. Then there is a unique solution $u(x, t) \in \mathscr{E}_{t}^{1}(K(\Omega))[0, T] \cap \mathscr{E}_{t}^{2}\left(L^{2}(\Omega)\right)[0, T]$ of the equation (2.3) satisfying

$$
u=u_{0}, u_{t}=v_{0} \quad \text { on } t=0 \text { (initial condition) }
$$

and

$$
B_{2} u=0 \quad \text { weakly on } \partial_{2} \Omega \times[0, T] \text {. }
$$

Theorem 2. In addition to the assumption of Theorem 1, assume that $u_{0} \in H_{\text {loc }}^{2}(\bar{\Omega}-S),{ }^{2)}$ where $S$ is the boundary of $\partial_{1} \Omega\left(\partial_{2} \Omega\right)$. Then $u(x, t)$ also belongs to $H_{\text {loc }}^{2}(\bar{\Omega}-S)$. Thus the solution satisfies $B_{2} u=0$

2) The space $H_{\mathrm{loc}}^{2}(\bar{\Omega}-S)$ is the set of functions belonging to locally $H^{2}$ in $\bar{\Omega}-S$. 
in the interior of $\partial_{2} \Omega$.

Remark. Here we have assumed (2.1). Hence if $\partial_{1} \Omega$ is an $(n-2)$ dimensional compact manifold, our theorem holds. With difference method, Babaeva and Namazov [1] has shown the existence of the solution for our problem also when $\partial_{2} \Omega$ is an $(n-2)$-dimensional compact manifold.

3. Let us consider the space $H=K(\Omega) \times L^{2}(\Omega)$ with the inner product

$$
\begin{aligned}
\left(U_{1}, U_{2}\right)_{H}= & \sum_{i, j}\left(a_{i j}(x) \frac{\partial u_{1}}{\partial x_{i}}, \frac{\partial u_{2}}{\partial x_{j}}\right)+\left(v_{1}, v_{2}\right) \\
& +\int_{\partial_{2} \Omega} \sigma(x) u_{1} \bar{u}_{2} d S+c_{1}\left(u_{1}, u_{2}\right),
\end{aligned}
$$

where $U_{i}=\left\{u_{i}, v_{i}\right\}(i=1,2)$ and $c_{1}$ is a sufficiently large constant depending only on $a_{i j}$ and $\sigma$. We denote by $\|U\|_{H}$ the $H$-norm of $U$. Obviously the space $H$ is complete and by the well-known interpolation relation (see e.g. [6]) the norm $\|U\|_{H}$ is equivalent to $\|u\|_{1}+\|v\|_{0}(U=$ $\{u, v\})$.

The formulation in this section is radically due to the book of H.G. Garnir. ${ }^{3)}$

Set the operator $A(t)$ in such a way that

$$
A(t)=\left(\begin{array}{cc}
0 & 1 \\
-L & -M
\end{array}\right),
$$

where $M=2 \sum_{i} h_{i}(x) \frac{\partial}{\partial x_{i}}+h(x) \quad$ (see (2.3)). Then $A(t)$ is a closed operator from $H$ to itself having the following definition domain

$$
\begin{aligned}
D(A(t))= & \left\{U=\{u, v\} \mid u, v \in K(\Omega), L u \in L^{2}(\Omega)\right. \\
& \text { and } U \text { satisfies the boundary condition }\left(B_{2}\right) \text { on } \partial_{2} \Omega \\
& \text { in the sense of Definition 2.2\}. }
\end{aligned}
$$

3) Les Problèmes aux Limites de la Physique Mathématique, Birkhäuser, 1958. 
Since $D(A(t))$ is independent of $t$, we write simply by $D(A)$.

Remark. Mizohata [5] and Ikawa [3] have set

$$
H=H_{0}^{1}(\Omega) \times L^{2}(\Omega)
$$

and

$$
D(A)=\left(H^{2}(\Omega) \cap H_{0}^{1}(\Omega)\right) \times H_{0}^{1}(\Omega)
$$

for the case of the Dirichlet type boundary condition. They have set also for the case of the Neumann type boundary condition as follows:

$$
H=H^{1}(\Omega) \times L^{2}(\Omega)
$$

and

$$
\begin{aligned}
D(A)=\left\{U=\{u, v\} \mid u \in H^{2}(\Omega), v \in H^{1}(\Omega)\right. \text { and } \\
\left.\qquad \frac{d}{d n} u-<h, \gamma>v+\sigma u=0 \text { on } \partial \Omega\right\} .
\end{aligned}
$$

Lemma 1. There is a positive constant $c_{2}$ depending only on $A(t)$ and $\sigma(x)$ such that it holds that for any $U \in D(A)$,

$$
\left|(U, A(t) U)_{H}+(A(t) U, U)_{H}\right| \leqq c_{2}^{\prime} \mid U \|_{H}^{2} .
$$

Proof. We easily see

$$
\begin{aligned}
(U, A(t) U)_{H}+(A(t) U, U)_{H} \\
=\sum_{i, j}\left(a_{i j} \frac{\partial u}{\partial x_{i}}, \frac{\partial v}{\partial x_{j}}\right)+(v,-L u-M v) \\
\quad+\int_{\partial_{2} \Omega} \sigma u \bar{v} d S+c_{1}(u, v) \\
\quad+\sum_{i, j}\left(a_{i j} \frac{\partial v}{\partial x_{i}}, \frac{\partial u}{\partial x_{j}}\right)+(-L u-M v, v) \\
\quad+\int_{\partial_{2} \Omega} \sigma v \bar{u} d S+c_{1}(v, u) .
\end{aligned}
$$

Since $U$ satisfies the boundary condition $\left(B_{2}\right)$ on $\partial_{2} \Omega$ (see Definition 2.2), we have

$$
\sum_{i, j}\left(a_{i j} \frac{\partial u}{\partial x_{i}}, \frac{\partial v}{\partial x_{j}}\right)+\int_{\partial_{2} \Omega} \sigma u \bar{v} d S
$$




$$
\begin{gathered}
=(L u, v)-\left(\sum_{i} b_{i} \frac{\partial u}{\partial x_{i}}+c u, v\right) \\
+\int_{\partial_{2} \Omega}<h, \gamma>v \bar{v} d S .
\end{gathered}
$$

Further it is easily seen that

$$
\begin{aligned}
(M v, v) & +(v, M v)=2 \int_{\partial_{2} \Omega}\langle h, \gamma\rangle v \bar{v} d S \\
& -2 \sum_{i}\left(\frac{\partial h_{i}}{\partial x_{i}} v, v\right)+(h v, v)+(v, h v) .
\end{aligned}
$$

Combining (3.4), (3.5) and (3.3), we have proved the lemma.

Lemma 2. If $\lambda$ is real and $|\lambda| \geqq c_{2}$, we have for any $U \in D(A)$

$$
\|(\lambda I-A(t)) U\|_{H} \geqq\left(|\lambda|-c_{2}\right)\|U\|_{H} .
$$

Proof. We easily see

$$
\|(\lambda I-A(t)) U\|_{H}^{2} \geqq \lambda^{2}\|U\|_{H}^{2}-\lambda\left\{(U, A(t) U)_{H}+(A(t) U, U)_{H}\right\} .
$$

By Lemma 1 we get

$$
\begin{aligned}
\|(\lambda I-A(t)) U\|_{H}^{2} \geqq\left(\lambda^{2}-|\lambda| c_{2}\right)\|U\|_{H}^{2} \\
\geqq\left\{\left(|\lambda|-c_{2}\right)^{2}+c_{2}\left(|\lambda|-c_{2}\right)\right\}\|U\|_{H}^{2} .
\end{aligned}
$$

Now set for any $\varphi, \phi \in K(\Omega)$

$$
\begin{aligned}
B_{t}[\varphi, \psi] & =\sum_{i, j}\left(a_{i j} \frac{\partial \varphi}{\partial x_{i}}, \frac{\partial \psi}{\partial x_{j}}\right) \\
& +\left(\sum_{i} b_{i} \frac{\partial \varphi}{\partial x_{i}}+c \varphi, \psi\right)+\int_{\partial_{2} \Omega} \sigma \varphi \bar{\psi} d S \\
& -\lambda \int_{\partial_{2} \Omega}<h, \gamma>\varphi \bar{\psi} d S \\
& +\lambda(M \varphi, \psi)+\lambda^{2}(\varphi, \psi) .
\end{aligned}
$$

Then using the interpolation relation for the trace of functions (see e.g. [6]), we see that there is a positive constant $c_{3}$ such that if $\lambda$ is real and $|\lambda| \geqq c_{3}$, it holds for any $\varphi \in K(\Omega)$,

$$
\left|B_{t}[\varphi, \varphi]\right| \geqq c_{3}^{-1}\|\varphi\|_{1}^{2}
$$


It is easily seen that

$$
\left|B_{t}[\varphi, \psi]\right| \leqq c_{4}\|\varphi\|_{1}\|\psi\|_{1} \text { for any } \varphi, \psi \in K(\Omega) .
$$

Hence by the theorem of Lax-Milgram we have the following

Lemma 3. For any given anti-linear functional $l$ on $K(\Omega)$ there is a unique solution $u \in K(\Omega)$ of the equation

$$
B_{t}[u, \varphi]=l(\varphi) \text { for any } \varphi \in K(\Omega) \text {. }
$$

From Lemma 3 we immediately see that

Lemma 4. If $\lambda$ is real and $|\lambda| \geqq c_{3}$, then for any $F \in H$ there is $a$ unique solution $U \in D(A)$ of the equation

$$
(\lambda I-A(t)) U=F \text {. }
$$

Proof. Put $U=\{u, v\}$ and $F=\{f, g\}$. Then the equation (3.7) is equivalent to

$$
v=\lambda u-f
$$

and

$$
L u+\lambda(\lambda+M) u=g+(\lambda+M) f .
$$

Let us put in Lemma 3

$$
l(\varphi)=((\lambda+M) f+g, \varphi)-\int_{\partial_{2} \Omega}<h, \gamma>f \bar{\varphi} d S
$$

Then $l$ satisfies the assumption of Lemma 3 by the well-known inequality. Thus there is a $u \in K(\Omega)$ such that $B_{t}[u, \varphi]=l(\varphi)$ for any $\varphi \in K(\Omega)$. In particular, taking $\varphi$ as in $C_{0}^{\infty}(\Omega)$, we see that (3.9) holds and $L u \in$ $L^{2}(\Omega)$. Hence we get from (3.6), (3.8) and (3.9)

$$
\begin{aligned}
& \sum_{i, j}\left(a_{i j} \frac{\partial u}{\partial x_{i}}, \frac{\partial \varphi}{\partial x_{j}}\right)+\int_{\partial_{2} \Omega} u \bar{\varphi} d S \\
& -\int_{\partial_{2} \Omega}<h, \gamma>v \bar{\varphi} d S \\
& =\left(-\left\{\sum_{i, j} \frac{\partial}{\partial x_{i}}\left(a_{i j} \frac{\partial}{\partial x_{j}}\right)\right\} u, \varphi\right) \text { for any } \varphi \in K(\Omega) .
\end{aligned}
$$


By Definition 2.2, this equality implies that $U$ satisfies the boundary condition $\left(B_{2}\right)$ on $\partial_{2} \Omega$. Thus $U \in D(A)$. Therefore we have completed the proof.

Let us rewrite by new $c_{2}$ the maximum of $c_{2}$ and $c_{3}$. Then combining Lemmas 2 and 4, we obtain

Lemma 5. If $|\lambda| \geqq c_{2}$, then it holds that

$$
\left\|(\lambda I-A(t))^{-1}\right\|_{H} \leqq \frac{1}{|\lambda|-c_{2}} .
$$

4. In this section we shall prove that $D(A)$ is dense in $H$. Let us denote by $C_{(0)}^{\infty}\left(R_{-}^{n}\right)$ the set of $C^{\infty}$ functions on $x_{n} \leqq 0$ having compact support there. Then we have

Lemma 6. For $u$ in $C_{(0)}^{\infty}\left(R_{-}^{n}\right)$ there is a sequence $\left\{\varphi_{i}\right\}$ in $C_{(0)}^{\infty}\left(R_{-}^{n}\right)$ such that

(i) $\varphi_{i} \rightarrow u$ in $H^{1}\left(R_{-}^{n}\right)$

(ii) $\quad \frac{\partial}{\partial x_{n}} \varphi_{i}=0 \quad$ on $x_{n}=0$

and

(iii) if $u\left(x^{\prime}, x_{n}\right)^{4)}=0$ for the fixed $x^{\prime}$ and any $x_{n}$, then each $\varphi_{i}\left(x^{\prime}, x_{n}\right)$ vanishes also for the $x^{\prime}$ and any $x_{n}$.

The proof of Lemma 6 is familiar, so it is sufficient to show construction of $\varphi_{i}$. The functions $\varphi_{i}$ are given as follows;

$$
\varphi_{i}\left(x^{\prime}, x_{n}\right)=\int_{-\infty}^{x_{n}} \alpha_{i}(s) \frac{\partial u}{\partial x_{n}}\left(x^{\prime}, s\right) d s
$$

where

$$
\alpha_{i}(s)=\left\{\begin{array}{lll}
0 & \text { if } & s>-\frac{1}{i} \\
1 & \text { if } & s<-\frac{2}{i} .
\end{array}\right.
$$

For the bounded function $\sigma\left(x^{\prime}\right)$ on $x_{n}=0$, let us take a new sequence 4) Put $x^{\prime}=\left(x_{1}, \ldots, x_{n-1}\right)$. 
$\left\{\varphi_{i} \exp \left(x_{n} \sigma\left(x^{\prime}\right)\right)\right\}$. Then we have also the following

Lemma 7. For $u$ in $C_{(0)}^{\infty}\left(R_{-}^{n}\right)$ there is a sequence $\left\{\varphi_{i}\right\}$ in $C_{(0)}^{\infty}\left(R_{-}^{n}\right)$ such that the properties (i), (iii) in Lemma 6 hold and in the place of (ii) the following property holds:

$$
\left(\frac{\partial}{\partial x_{n}}+\sigma\left(x^{\prime}\right)\right) \varphi_{i}=0 \quad \text { on } x_{n}=0
$$

Generalizing Lemma 7, we prove the following

Lemma 8. For any $u \in K(\Omega)$, there is a sequence $\left\{\varphi_{i}\right\} \subset C^{\infty}(\bar{\Omega})$ satisfying $\left(\frac{d}{d n}+\sigma(x)\right) \varphi_{i}=0$ on $\partial_{2} \Omega$ such that each $\varphi_{i}$ vanishes in a neighborhood of $\overline{\partial_{1} \Omega}$ and $\varphi_{i} \rightarrow u$ in $H^{1}(\Omega)$.

Proof. We may assume that $u$ is in $C^{\infty}(\bar{\Omega})$ and $u=0$ in a neighborhood of $\overline{\partial_{1} \Omega}$. For each point $P$ in $\bar{\Omega}$ let us take an open neighborhood $U(P)$ in such a way that

$$
\begin{array}{ll}
\overline{U(P)} \subset \Omega & \text { for } P \in \Omega, \\
u=0 \text { in } U(P) & \text { for } P \in \overline{\partial_{1} \Omega}
\end{array}
$$

and

$$
\overline{U(P)} \cap \overline{\partial_{1} \Omega}=\phi \quad \text { for } P \in \partial_{2} \Omega .
$$

Since (2.1) holds from our assumption, such a selection of $U(P)$ is possible.

Now there is a finite point set $\left\{P_{1}, \ldots, P_{N}\right\}$ and the union of $U\left(P_{k}\right)$ $(1 \leqq k \leqq N)$ covers $\bar{\Omega}$. Let the function $\alpha_{k}$ be in $C_{0}^{\infty}\left(U\left(P_{k}\right)\right)$ such that $\sum_{k=1}^{N} \alpha_{k} \equiv 1$ in $\Omega$. We assume that the points $P_{1}, \ldots, P_{N^{\prime}}\left(N^{\prime} \leqq N\right)$ are in $\partial_{2} \Omega$. Each subdomain $\overline{U\left(P_{k}\right) \cap \Omega}\left(1 \leqq k \leqq N^{\prime}\right)$ can be mapped in a one to one $C^{\infty}$ way into $y_{n} \leqq 0^{5)}$ such that theo perator $\frac{d}{d n}$ on $U\left(P_{k}\right)$ is transformed into $\frac{\partial}{\partial y_{n}}$. Applying Lemma 8 for $\alpha_{k} u$ on $y_{n} \leqq 0$, we can find a sequence $\left\{\varphi_{i}^{(k)}\right\}$ $C C^{\infty}(\bar{\Omega})\left(1 \leqq k \leqq N^{\prime}\right)$ having the following property that

5) We denote by $\left(y_{1}, \ldots, y_{n}\right)$ the new coordinate. 


$$
\varphi_{i}^{(k)}=0 \text { in a neighborhood of } \overline{\partial_{1} \Omega},
$$

$$
\left(\frac{d}{d n}+\sigma(x)\right) \varphi_{i}^{(k)}=0 \quad \text { on } \partial_{2} \Omega
$$

and

$$
\varphi_{i}^{(k)} \rightarrow \alpha_{k} u \quad \text { in } H^{1}(\Omega)
$$

Setting

$$
\varphi_{i}=\sum_{k=1}^{N^{\prime}} \varphi_{i}^{(k)}+\sum_{k=N^{\prime}+1}^{N} \alpha_{k} u
$$

we easily see

$$
\varphi_{i} \rightarrow u \text { in } H^{1}(\Omega) .
$$

The other properties of $\left\{\varphi_{i}\right\}$ is obvious from (4.1) and (4.2). Hence we have finished the proof.

Finally we have

Lemma 9. The definition domain $D(A)$ (see (3.2)) is dense in $H$.

Proof. Let the vector function $\{u, v\}$ be in $H\left(=K(\Omega) \times L^{2}(\Omega)\right)$. First we take a sequence $\left\{v_{i}\right\} \subset C_{0}^{\infty}(\Omega)$ converging to $v$ in $L^{2}(\Omega)$. Secondly we set $u_{i}=\varphi_{i}$ for the sequence $\left\{\varphi_{i}\right\}$ in Lemma 8. Obviously, $\left\{u_{i}, v_{i}\right\} \rightarrow$ $\{u, v\}$ in $H$. Since $\left(\frac{d}{d n}+\sigma(x)\right) u_{i}=0$ on $\partial_{2} \Omega$, we see that (2.7) holds by Green's formula. Thus each $\left\{u_{i}, v_{i}\right\}$ satisfies the boundary condition $\left(B_{2}\right)$ on $\partial_{2} \Omega$. Hence $D(A)$ is dense in $H$.

5. In virtue of Lemma 5 and 9 , we can apply the theory of evolution equations quite similarly as in [3] and [5] as follows. Suppose that $F(t)=\{0, f(t)\}$ is in $D(A)$ and $F(t), A F(t) \in \mathscr{E}_{t}^{0}(H)[0, T]$. Then for any given $U_{0}=\left\{u_{0}, v_{0}\right\} \in D(A)$, there is a unique solution $U(t)=\{u(t), v(t)\}$ $\in D(A) \cap \mathscr{E}_{t}^{1}(H)[0, T]$ of the equation

$$
\frac{d}{d t} U(t)=A U(t)+F(t) \quad \text { in } 0<t \leqq T
$$

with the initial condition $U(0)=U_{0}$. The equation (5.1) is equivalent to 
(2.3). Since $v=u_{t}$ and (2.7) holds, we see that $u$ satisfies the boundary condition (2.5) weakly on $\partial_{2} \Omega \times[0, T]$ (see Definition 2.1). Hence Theorem 1 in Section 2 has been shown.

The statement of Theorem 2 is proved quite similarly as in Theorem 1 , if we add to the definition domain $D(A)$ the condition that $u \in H_{\text {loc }}^{2}$ $(\bar{\Omega}-S)$.

Finally, we show the energy inequality for $U(t) \in D(A) \cap \mathscr{E}_{t}^{1}(H)[0, T]$. It is easily seen that from Lemma 1

$$
\begin{aligned}
\frac{d}{d t} & \|U(t)\|_{H}^{2}=\left(U^{\prime}(t), U(t)\right)_{H}+\left(U(t), U^{\prime}(t)\right)_{H} \\
& =(A U(t)+F(t), U(t))_{H}+(U(t), A U(t)+F(t))_{H} \\
& \leqq 2 c_{2}\|U(t)\|_{H}^{2}+2\|U(t)\|_{H}\|F(t)\|_{H} .
\end{aligned}
$$

From this it follows

$$
\|U(t)\|_{H} \leqq e^{c_{2} t}\left(\|U(0)\|_{H}+\int_{0}^{t}\|F(s)\|_{H} d s\right) .
$$

Hence

$$
\|u(t)\|_{1}+\| u^{\prime}(t) !_{0} \leqq C e^{c_{2} t}\left(\|u(0)\|_{1}+\|v(0)\|_{0}+\int_{0}^{t}\|f(s)\|_{0} d s\right)
$$

\section{References}

[1] Babeava, A.A. and G.K. Namazov, Hyperbolic equations with discontinuous coefficients, degenerate on the initial plane, Izv. Akad. Nauk Azerbaidzan SSSR Ser. Fiz. - Tehn. Mat. Nauk, no. 5 (1967), 11-16 (Russian).

[2] Čehlov, V.I., A mixed problem with discontinuous boundary conditions for the wave equation, Soviet Math. Dokl. 9 (1968), 1472-1475.

[3] Ikawa, M., Mixed problems for hyperbolic equations of second order, J. Math. Soc. Japan, 20 (1968), 580-608.

[4] Lions, J.L., Une remarque sur les applications du théorèmes de Hille-Yosida, $J$. Math. Soc. Japan, 9 (1957), 62-70.

[5] Mizohata, S., Quelque problèmes au bord, du type mixte, pour des équations hyperboliques, Séminaire sur les équations aux derivées partielles, Collèdge de France, 1966-1967, 23-60.

[6] Martial Differential Equations, Iwanami, 1965 (Japanese).

[7] Yosida, K., On operator theoretical integration of wave equations, J. Math. Soc. Japan, 8 (1956), 79-92. 
